\title{
Selection of high affinity ligands to hepatitis B core antigen from a phage-displayed cyclic peptide library.
}

\begin{abstract}
M13 phages that display random disulfide constrained heptapeptides on their gpIII proteins were used to select for high affinity ligands to hepatitis B core antigen ( $\mathrm{HBcAg}$ ). Phages bearing the amino acid sequences C-WSFFSNI-C and C-WPFWGPW-C were isolated, and a binding assay in solution showed that these phages bind tightly to full-length and truncated $\mathrm{HBcAg}$ with KDrel values less than $25 \mathrm{nM}$, which is at least 10 orders of magnitude higher than phage carrying the peptide sequence LLGRMK selected from a linear peptide library. Both the phages that display the constrained peptides were inhibited from binding to $\mathrm{HBcAg}$ particles by a monoclonal antibody that binds specifically to the immunodominant region of the particles. A synthetic heptapeptide with the amino acid sequence WSFFSNI derived from one of the fusion peptides inhibits the binding of large surface antigen (L-HBsAg) to core particles with an IC50 value of $12 \pm 2 \mu \mathrm{M}$. This study has identified a smaller peptide with a greater inhibitory effect on L-HBsAg-HBcAg association.
\end{abstract}

Keyword: Biopanning; Dissociation constant; Filamentous phage; HBV assembly; Inhibition study 